\title{
Psychosocial well-being over the two years following cardiac rehabilitation initiation \& association with heart-health behaviors
}

\author{
Saba Ali ${ }^{\mathrm{a}}$, Carolina Santiago de Araújo Pio ${ }^{\mathrm{a}}$, Gabriela S.S. Chaves ${ }^{\mathrm{a}, \mathrm{b}}$, Raquel Britto ${ }^{\mathrm{b}}$, \\ Robert Cribbie ${ }^{\mathrm{a}}$, Sherry L. Grace ${ }^{\mathrm{a}, \mathrm{c}, \mathrm{d}, *}$ \\ ${ }^{\text {a }}$ York University, Toronto, Ontario, Canada \\ ${ }^{\mathrm{b}}$ Universidade Federal de Minas Gerais, Belo Horizonte, Brazil \\ ${ }^{\mathrm{c}}$ University Health Network, Toronto, Ontario, Canada \\ ${ }^{\mathrm{d}}$ University of Toronto, Toronto, Ontario, Canada
}

\section{A R T I C L E I N F O}

\section{Keywords:}

Cardiac rehabilitation

Psychology

Exercise

Heart disease

\begin{abstract}
A B S T R A C T
Objective: To track psychosocial well-being over 2 years following cardiac rehabilitation (CR) initiation, and its' association with heart-health behaviors.

Methods: Patients from $3 \mathrm{CR}$ programs were approached at their first visit, and consenters completed a survey. Participants were emailed surveys again 6 months, 1 and 2 years later. Depressive symptoms (PHQ-8) and quality of life were assessed at each point, as were exercise, nutrition, smoking and medication adherence, among other well-being indicators.

Results: Of 411 participants, $46.7 \%$ were retained at 2 years. Post-CR, there was $70 \%$ concordance between participants' desired and actual work status. Depressive symptoms were consistently minimal over time (mean $=3.17 \pm 0.37$ ); Quality of life was high, and increased over time $(p=.01)$. At 2 years, $56.9 \%$ participants met exercise recommendations, and 5.4\% smoked. With adjustment, greater self-regulation was associated with significantly greater exercise at intake; greater exercise self-efficacy was significantly associated with greater exercise at 1 year; greater disease management self-efficacy was significantly associated with greater exercise at 2 years; greater environmental mastery (actual) was significantly associated with greater exercise at 2 years. Lower depressive symptoms were significantly associated with better nutrition at 2 years.

Conclusion: CR initiators are thriving, and this relates to better exercise and diet.
\end{abstract}

\section{Introduction}

Cardiovascular disease (CVD) is one of the most prevalent health conditions worldwide [1]. Upwards of one in five cardiac patients suffers from depression; this is a much higher burden than is observed in the general population [2]. This depression, among other psychosocial factors such as social isolation, anxiety and hostility, is associated with higher mortality and morbidity in cardiac patients [3]. Indeed depression is recognized as a risk factor for poor prognosis in these patients by the American Heart Association [4]. Yet, it may be natural to experience psychological distress in response to a cardiac event, and there are data to suggest it wanes in many patients with time [5] and that some patients may even make meaning in the life-threatening cardiac experience and find benefit [6]. However, little is known about the trajectory of psychosocial status over the long-term in cardiac patients.

Indeed there has been recent interest in psychosocial well-being in cardiac patients, or positive cognitive, social and emotional states or traits, such as purpose in life and positive affect [7], as well as role functioning. Reviews have demonstrated cardiac patients with greater well-being have better outcomes, including reduced hospital readmissions, lower mortality [8] and reduced risk of recurrent cardiovascular events [9]. These outcomes are observed even after taking into consideration psychological distress such as depression. The mechanisms for these associations include physiological factors (e.g., inflammation), and also likely greater engagement in heart-health behaviors [8-14].

However, the extent to which patients experience psychosocial wellbeing, and hence may reap some of these major health benefits, is not well characterized. Using the Broaden and Build theory (Fig. 1) [15], the objective of this study was to characterize psychosocial well-being over 2 years following a cardiac event or procedure. This theory posits that positive emotions broaden one's awareness, which results in a broadened behavioral repertoire (including taking action or building

\footnotetext{
* Corresponding author at: York University, Bethune 368, 4700 Keele Street, Toronto, ON M3J 1P3, Canada.

E-mail address: sgrace@yorku.ca (S.L. Grace).
} 


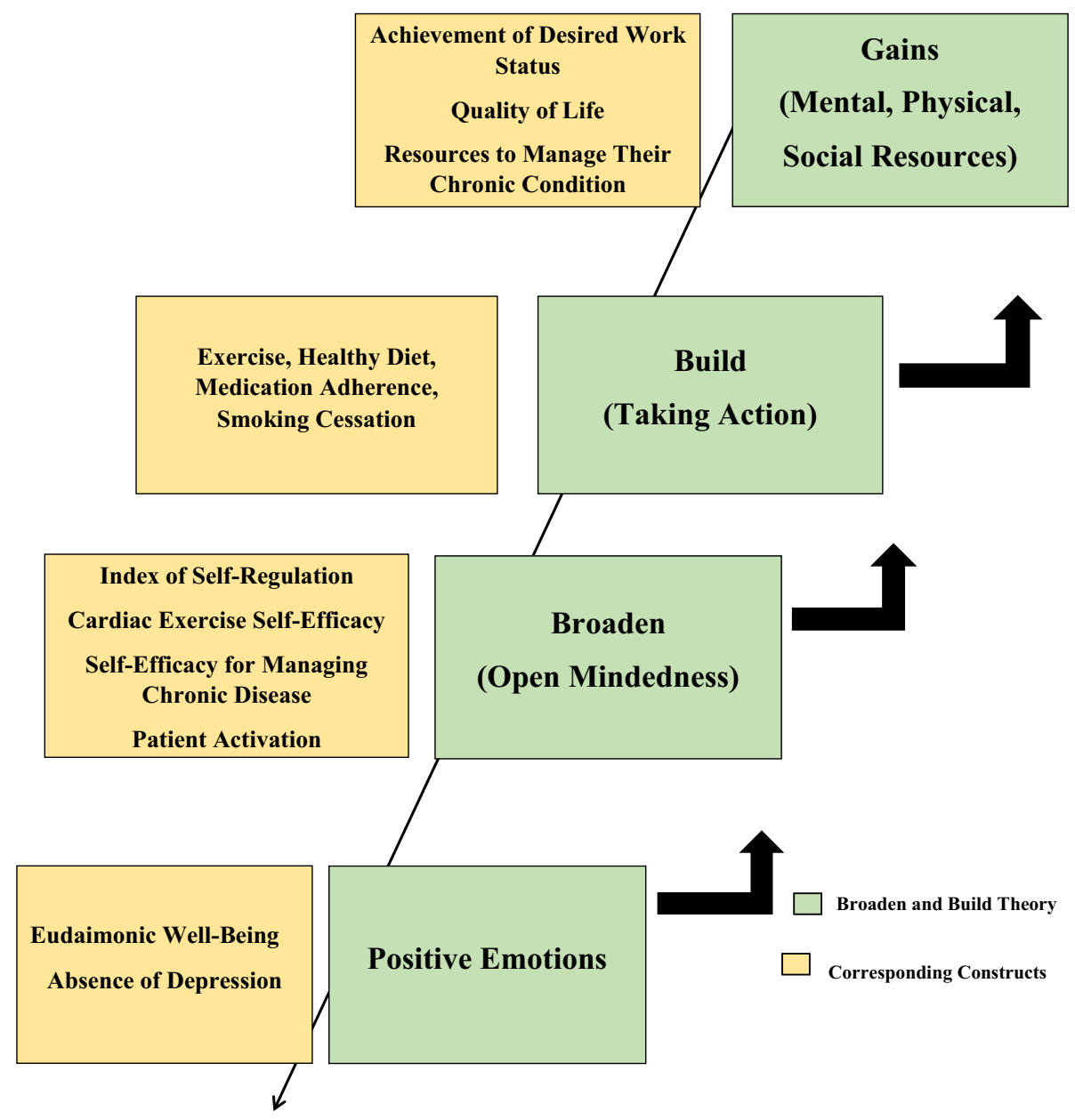

Fig. 1. Broaden and Build theory juxtaposed with corresponding study constructs.

improved heart-health behaviors); this in turn results in gains (e.g., improved quality of life) and increases in resources (physical and social). Specifically, with regard to positive emotions, eudaimonic wellbeing was assessed and depressive symptoms were tracked. With regard to broadening, patient activation, self-regulation, as well as self-efficacy for exercise and chronic disease management were considered. With regard to building, the association of these psychosocial well-being indicators with heart-health behaviors was investigated, namely, physical activity, smoking, diet and medication adherence. It was hypothesized that more positive psychosocial well-being would be significantly associated with better heart-health behaviors. Finally, with regard to gains, achievement of desired work status, and quality of life (QoL) were characterized.

\section{Methods}

\subsection{Design and procedure}

Herein, secondary analysis of a cardiac rehabilitation (CR) program evaluation is presented. This study was observational, and prospective in design. Approval was received from the research ethics review boards at the institutions of each participating CR site.

Patients referred to $\mathrm{CR}$ at one of the study centers following a qualifying hospitalization or diagnosis were approached to participate at their initial visit (generally an intake assessment) between July 2010 and February 2014. Written, informed consent was solicited by administrative staff at the site. Based on median times from hospital discharge to CR initiation in the region [16], the baseline assessment would have occurred approximately 1.5 months post-event/procedure.
Participants were provided a self-administered survey in paper format to take home, or emailed a link to complete the survey online at home. The survey assessed sociodemographic characteristics including work status, self-regulation, depression and QoL. The latter 2 scales were administered at all assessment points.

A second survey was provided to all study participants from the central study office (regardless of CR program completion), via mail and/or online. It assessed work status, and desired work status. It was administered to correspond with CR program duration (this varied somewhat by site; details reported elsewhere) [17]. For all participants CR completion was ascertained from program charts (Fig. 2).

Finally, a survey was also administered to all study participants (again regardless of CR completion) at 1 and 2 years' post-intake. The former included exercise self-efficacy, the latter included self-efficacy for managing chronic disease, patient activation and eudaimonic wellbeing.

Physical activity, nutrition, medication adherence and smoking status were also assessed at all 4 points. Timing of assessments at CR admission, to correspond with CR discharge, and at 1 and 2 years from CR admission is depicted in Fig. 2. To optimize the response rate, at each assessment point, non-responders were sent a repeat e-mail, and then if they still had not responded they were contacted by telephone.

\subsection{Participants}

The cohort consisted of participants from $3 \mathrm{CR}$ sites in the Greater Toronto Area, Canada and one satellite program. This convenience sample consisted of all consenting participants attending an initial visit at 1 of these programs. Participants were referred to the CR programs 


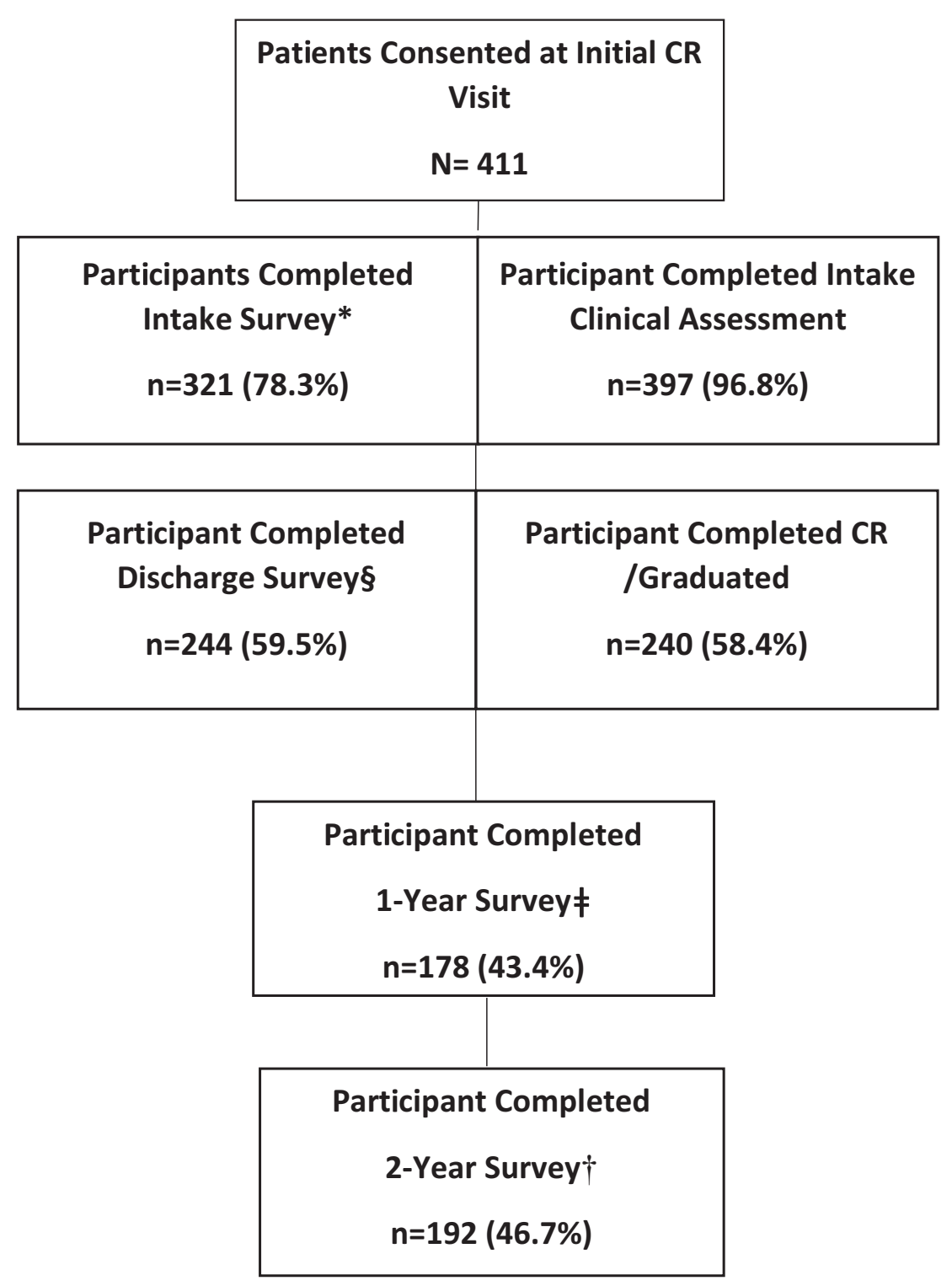

Fig. 2. Participant flow diagram with assessment points

$\mathrm{CR}=$ cardiac rehabilitation.

*Depressive symptoms and quality of life, as well as 4 health behaviors assessed in all 4 surveys.

§Actual and desired work status assessed at intake and discharge.

$\neq$ Exercise self-efficacy assessed.

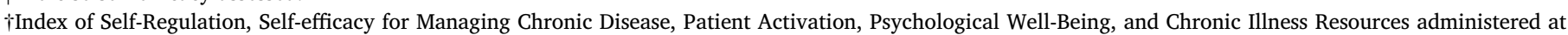
this assessment.

Note: boxes on right denote data ascertained from CR charts.

with the following cardiac diagnoses or procedures: acute coronary syndrome, chronic stable angina, or stable heart failure, as well as percutaneous coronary or valvular intervention, coronary artery bypass graft $(C A B G) \pm$ valve surgery, cardiac transplantation, or mild nondisabling stroke [18]. The inclusion criterion for the larger program evaluation was that participants were deemed eligible to complete $\mathrm{CR}$ upon initial assessment (i.e. no comorbidities identified or indications from the graded stress test that would preclude exercise training). Participants who were not proficient in the English language were excluded from the study.

\subsection{Measures}

Sociodemographic characteristics such as participants' ethnocultural background (adapted from Statistics Canada categorizations), marital status, and highest educational attainment, were assessed via self-report through forced choice response options. Participants were also asked to report if they were limited in paid employment due to their CVD. Clinical data were extracted from CR referral forms, as well as CR intake and discharge assessments, where available. To assess psychosocial well-being, several measures were selected, based on administration in other, or relevance to, cardiac samples, as well as psychometric rigor. The manner in which they correspond to the Broaden and Build theory [15] is outlined below.

\subsubsection{Positive emotions}

The Patient Health Questionnaire-8 [19] is a reliable and validated depressive symptom screening scale, through which respondents are asked to report the frequency of depressed mood in the last 2 weeks. Each item is scored on a Likert-type scale from 0 (not at all) to 3 (nearly 
every day). A total score is computed by summing responses, with higher scores indicating more severe depressive symptoms. A score of $<5$ is considered indicative of minimal depressive symptoms (we concede this is not synonymous with lack of negative emotion or presence of positive emotion however). This was administered at all 4 assessment points.

The Ryff Scale of Psychological Well-Being is an 18-item [20], theoretically-based, validated measure that assesses multiple aspects of eudaimonic well-being. The measure assesses 6 domains, namely: (a) self-acceptance, (b) autonomy, (c) environmental mastery, (d) personal growth, (e) positive relationships, and (f) purpose in life. Respondents rate statements on a scale from 1 to 6 , with 1 indicating they "strongly disagree" and 6 indicating they "strongly agree". They are then asked to re-rate each item, however this time indicating an ideal score for each. Negatively-phrased items are reverse-coded, and subscales items are summed together to compute total scores. For each subscale, a higher score indicates the respondent has mastery in that area, whereas a lower score in a category indicates the struggle with the particular aspect of psychological well-being. This was administered at the 2-year follow-up.

\subsubsection{Broaden}

The Index of Self-Regulation [21] is a 9-item scale which measures the degree of an individuals' health behavior self-management, in the domains of reconditioning, stimulus control and behavioral monitoring. Responses to each of the items are scored on a 5 point scale ranging from 1 to 5 (strongly disagree to strongly agree). A mean is computed, with higher scores indicating greater use of self-regulatory strategies. This scale was administered in the initial survey only.

The Cardiac Exercise Self-Efficacy Instrument (CESEI) [22] is a 16item measure that assesses an individual's confidence in their ability to practice good physical activity habits. Items are rated on a 5-point Likert scale $(1=$ very little, $5=$ quite a lot); Higher scores indicate greater self-efficacy. This was administered at the 1 year follow-up.

The Self-Efficacy for Managing Chronic Disease [23] is a 6-item scale assessing emotional functioning, role function, communicating with physician and symptom control. Each of the items is scored on a 10-point Likert scale (not at all confident to totally confident). The total score is computed by calculating the mean of the items; higher scores indicate higher self-efficacy. This was administered at the 2 year followup.

The Patient Activation Measure (PAM)-13 [24] assesses the knowledge, skills and confidence necessary to manage one's health and health care. Responses to each of the 13 items are scored on a 4-point scale ranging from 1 to 4 (strongly agree to strongly disagree). Based on responses, a raw score for each participant is derived, which corresponds to 1 of 4 stages of activation. Raw scores between 67.1 and 100 correspond to stage 4 , which suggests respondents take an active role with regards to their own health. Raw scores ranging between 55.2 and 67.0 correspond to stage 3 , which reflects respondents have the confidence and knowledge to take action regarding their health. Raw scores ranging from 47.1-55.1 correspond to stage 2, which suggests respondents lack basic knowledge and experience or success with health behavior change. Finally, raw scores between 0 and 47 correspond to stage 1 , which indicates that respondents view their doctor as the one in charge and so do not feel in control of their own health. This was administered at the 2 year follow-up.

\subsubsection{Build}

Physical activity, nutrition and medication adherence were assessed via psychometrically-validated scales. Participants were asked to selfreport their smoking status (current, former or never). These were administered at all 4 assessment points.

The Godin Leisure-Time Exercise Questionnaire [25] is a brief and reliable instrument to assess usual physical activity during a typical 1week period. Frequencies of strenuous, moderate, and light-intensity activities were assessed, and weighted. Higher scores indicated a greater amount of exercise. Those scoring above 24 were considered to be sufficiently active and meeting the recommended guidelines of $>$ 150 min of moderate to vigorous intensity exercise per week.

The Health Promoting Lifestyle Profile II [26] nutrition subscale contains 6 statements that assess daily personal nutrition habits. Response options range from 1 (never) to 4 (routinely), indicating the frequency with which a particular nutrition behavior is practiced. A mean value was computed, with higher scores representing a healthier diet. Finally, the 4-item version of Morisky's Medication Adherence Scale [27] was also administered. Scoring information is available from the author.

\subsubsection{Gains}

The EuroQoL-5D-3L (quality of life; EQ) is a standardized instrument that measures generic health status [28]. Respondents are asked to indicate their QoL in 5 dimensions (e.g., mobility, self-care items assessing mental and physical resources), by choosing one of the following response options: (1) no problems, (2) some problems, (3) extreme problems. Responses are then converted into a single utility score by applying a formula that attaches values (weights) to each response option. Higher utility scores (closer to 1.0) indicate better QoL. The EQ Visual Analogue Scale (VAS) was also administered, through which respondents rated their QoL on a vertical scale, with one end point being the "Best Imaginable Health State" with a score of 100 and the other being the "Worst Imaginable Health State" with a score of 0 . Both scales were administered at all 4 assessment points.

Social resources were assessed in 2 ways. First, the brief Chronic Illness Resource Survey [29] is a 29-item scale which measures support and resources in 7 areas: doctor and health care team, family and friends, personal, neighborhood/community, media/policy, organization and work. Participants were asked to rate the degree to which each resource/item was used over the past 6 months, on a Likert scale from 1 (not at all) to 5 (a great deal). Items are averaged, with higher scores indicating greater resources in a given domain. This was administered at the 2-year follow-up. Finally, participants were asked to self-report their actual and desired work status on the first 2 surveys.

\subsection{Statistical analysis}

SPSS software version 24 (IBM, Armonk, NY) was used for statistical analysis. Given the multiple comparisons, a significance cut-off value of $p<.01$ was applied throughout. Descriptive statistics were computed to describe the sociodemographic and clinical characteristics of the sample.

A descriptive examination of the psychosocial well-being constructs at each time point was performed. All analyses included only those who completed the assessments at the given time point(s) under testing. Paired $t$-tests were used to compare actual versus ideal psychological well-being. Congruency between desired versus actual work status was compared using Cohen's kappa. The trajectory of depressive symptoms and QoL were explored over the 2 years using general linear models.

Pearson's correlations were computed to assess the associations between psychosocial well-being indicators and heart-health behaviors, except the association with smoking was assessed using analysis of variance (with post-hoc LSD tests where significant). Finally, the significant associations were tested general linear models with adjustment for age, sex and sociodemographic and clinical characteristics which differed between retained participants versus those lost to follow-up. The psychosocial well-being indicators were the dependent variables (one model per indicator), and the heart-health behaviors were the independent variables (all 4 included in each model). 
Table 1

Participant characteristics at baseline $(N=411)$.

\begin{tabular}{|c|c|}
\hline Characteristic & $n(\%)$ or mean $\pm \mathrm{SD}$ \\
\hline \multicolumn{2}{|l|}{ Sociodemographic } \\
\hline $\mathrm{Age}^{\mathrm{a}}($ mean years $\pm \mathrm{SD})$ & $64.45 \pm 10.41$ \\
\hline $\operatorname{Sex}^{\mathrm{a}}(\%$ male $)$ & $287(70.2)$ \\
\hline Ethnicity (\% North American) & $126(39.9)$ \\
\hline Marital status (\% married) & $236(74.7)$ \\
\hline Education (\% completed < college/university) & $160(50.6)$ \\
\hline Work status (\% retired) & 145 (45.9) \\
\hline \multicolumn{2}{|l|}{ Clinical } \\
\hline Previous cardiac diagnosis ${ }^{\mathrm{b}}$ (\% yes) & $39(9.5)$ \\
\hline Peak METs $^{c}($ mean \pm SD) & $7.17 \pm 2.94$ \\
\hline \multicolumn{2}{|l|}{$\mathrm{CR}_{\text {referral indication }}{ }^{\mathrm{a}}$} \\
\hline PCI & $153(39.8)$ \\
\hline CABG & 109 (28.5) \\
\hline Other & $211(80.5)$ \\
\hline \multicolumn{2}{|l|}{ Risk factors $^{\mathrm{a}}$} \\
\hline Dyslipidemia & $293(82.5)$ \\
\hline Hypertension & $290(76.9)$ \\
\hline Obesity & $154(42.4)$ \\
\hline Diabetes & $77(21.8)$ \\
\hline Current smoker ${ }^{\mathrm{b}}$ & $14(4.5)$ \\
\hline
\end{tabular}

Note: Due to missing data, percentages for each characteristic were computed using a denominator value specific to the sample size with complete data for that variable.

SD, standard deviation; CABG, Coronary artery bypass grafting; PCI, Percutaneous Coronary Intervention; MET, Metabolic Equivalent of Task.

a Source is medical chart (hospital or cardiac rehabilitation program).

b Source is medical chart and/or self-report.

c From pre-cardiac rehabilitation graded exercise stress test.

\section{Results}

\subsection{Respondents characteristics}

Fig. 2 displays the flow of participants through the study. As shown, 321 participants completed the initial survey, and clinical data from the CR intake assessment could be extracted for 397 participants. Subsequently, there were 240 (58.4\%) participants that completed CR and hence had clinical data available for extraction post-program. Two hundred and forty-four participants completed the survey mailed at the time to correspond with the end of CR (of which 170 [69.7\%] completed CR). Finally, 178 and 192 participants respectively completed the 1 and 2-year post-intake survey via mail, of which $124(69.7 \%)$ and 125 (65.1\%) had previously completed CR respectively.

Table 1 displays participant sociodemographic and clinical characteristics at baseline. As shown, 3/4 s of participants were partnered, although relationship quality was not considered. Pre-CR, participants could on average achieve 7 metabolic equivalents of task (METs), which suggests they could live independently and undertake activities of daily living.

As reported elsewhere [30], there were some differences in the characteristics of participants retained at 2 years $(N=192)$ versus those lost-to-follow-up. With regard to sociodemographic characteristics, retained participants were more likely to self-report "North American" ethnocultural background compared to those lost to followup. With regard to clinical characteristics, retained participants were significantly less likely to have been referred to CR for revascularization compared to those lost to follow-up. With regard to psychosocial and health behavior constructs assessed at baseline, those retained at

Table 2

Psychosocial well-being indicators by assessment point.

\begin{tabular}{|c|c|c|c|}
\hline $\begin{array}{l}\text { Assessment point } \\
\text { Indicator (Broaden and Build construct) [15] }\end{array}$ & Mean $\pm \mathrm{SD} / n(\%)$ & Score range & Mean scores in comparative sample \\
\hline \multicolumn{4}{|l|}{ Intake } \\
\hline Index of self-regulation (Broaden) & $4.14 \pm 0.59$ & $1-5$ & $4.63[31]$ \\
\hline 1. Reconditioning & $4.35 \pm 0.61$ & $1-5$ & \\
\hline 2. Stimulus control & $4.02 \pm 0.72$ & $1-5$ & \\
\hline 3. Behavioral monitoring & $4.04 \pm 0.73$ & $1-5$ & \\
\hline \multicolumn{4}{|l|}{1 Year } \\
\hline Exercise self-efficacy (Broaden) & $3.83 \pm 0.92$ & $1-5$ & $4.11 \pm 0.63[22]$ \\
\hline \multicolumn{4}{|l|}{2 Years } \\
\hline Self-efficacy for managing chronic disease (Broaden) & $7.74 \pm 1.97$ & $1-10$ & $5.2 \pm 2.2[23]$ \\
\hline Patient activation (Broaden) & $67.55 \pm 18.73$ & $0-100$ & $56.6 \pm 12.9[32]$ \\
\hline 1- Disengaged and overwhelmed & $19(10.6 \%)$ & $\mathrm{n} / \mathrm{a}$ & $18.0 \%$ \\
\hline 2- Becoming aware but still struggling & $20(11.1 \%)$ & $\mathrm{n} / \mathrm{a}$ & $29.1 \%$ \\
\hline 3- Taking action & $64(35.6 \%)$ & $\mathrm{n} / \mathrm{a}$ & $35.7 \%$ \\
\hline 4- Maintaining behaviors and pushing further & $77(42.8 \%)$ & $\mathrm{n} / \mathrm{a}$ & $17.2 \%$ \\
\hline \multicolumn{4}{|l|}{ psychological well-being (positive emotions) } \\
\hline Environmental mastery (actual) & $14.57 \pm 2.54$ & $3-18$ & $14.9 \pm 2.8[20]$ \\
\hline Environmental mastery (ideal) & $15.04 \pm 2.22$ & $3-18$ & \\
\hline Autonomy (actual) & $13.74 \pm 2.18$ & $3-18$ & $15.2 \pm 2.2$ \\
\hline Autonomy (ideal) & $13.42 \pm 2.69$ & $3-18$ & \\
\hline Self-acceptance (actual) & $14.16 \pm 2.52$ & $3-18$ & $14.6 \pm 3.1$ \\
\hline Self-acceptance (ideal) & $13.44 \pm 3.21$ & $3-18$ & \\
\hline Positive relations (actual) & $14.05 \pm 2.90$ & $3-18$ & $14.8 \pm 3.2$ \\
\hline Positive relations (ideal) & $10.99 \pm 5.09$ & $3-18$ & \\
\hline Purpose in life (actual) & $12.84 \pm 2.95$ & $3-18$ & $14.4 \pm 3.2$ \\
\hline Purpose in life (ideal) & $11.48 \pm 4.30$ & $3-18$ & \\
\hline Personal growth (actual) & $14.36 \pm 2.27$ & $3-18$ & $15.7 \pm 2.5$ \\
\hline Personal growth (ideal) & $11.15 \pm 5.07$ & $3-18$ & \\
\hline Chronic illness resources (gains) & $2.90 \pm 0.61$ & $1-5$ & $2.8 \pm 0.6[33]$ \\
\hline Physician/healthcare team & $3.65 \pm 0.99$ & $1-5$ & $3.4 \pm 1.1$ \\
\hline Family and friends & $2.93 \pm 0.97$ & $1-5$ & $2.5 \pm 0.9$ \\
\hline Personal & $3.58 \pm 0.87$ & $1-5$ & $3.4 \pm 1.0$ \\
\hline Neighborhood & $2.58 \pm 1.00$ & $1-5$ & $2.2 \pm 0.9$ \\
\hline Organizations & $2.13 \pm 1.01$ & $1-5$ & $2.2 \pm 1.1$ \\
\hline Work & $3.18 \pm 1.23$ & $1-5$ & $1.8 \pm 0.9$ \\
\hline Community & $2.59 \pm 0.91$ & $1-5$ & $2.2 \pm 0.9$ \\
\hline
\end{tabular}

n/a, not applicable; SD, standard deviation. 
2 years had significantly lower depressive symptom scores $(p<.01)$ and significantly greater QoL $(p<.01)$ at intake. Those retained also engaged in significantly more exercise $(p=.001)$ and ate a healthier diet $(p<.05)$ at intake than those lost to follow-up; there were no differences in medication adherence or smoking. Finally, those who completed the 2 year follow-up survey were significantly more likely to have completed CR $(n=125,52.1 \%)$ than those who did not $(n=67$, 29.9\%; $p<.001)$.

\subsection{Positive emotions}

Psychosocial indicators are reported in Table 2, and juxtaposed in relation to mean scores in comparable samples in the published literature [20,22,23,31-33]. With regard to psychological well-being, scores were highest for environmental mastery and personal growth, and lowest for purpose in life. Participants desired significantly more environmental mastery than they felt (paired $t=3.68, p<.01$ ), but felt significantly more personal growth (paired $t=-8.04, p<.001$ ), positive relations with others (paired $t=-8.12, p<.001$ ), purpose in life (paired $t=-3.92, p<.001$ ), and self-acceptance (paired $t=-2.75, p<.05)$ than was their ideal.

Fig. 3A displays depressive symptoms across the 2 years in the retained sample. At intake, $132(55.7 \%)$ participants reported minimal depressive symptoms (i.e., PHQ < 5), with 105 (60.3\%) at 6 months, 80
(62.0\%) at 1 year and $84(57.5 \%)$ at 2 years reporting minimal symptoms at most. As shown, there were no significant changes in depressive symptoms over time.

\subsection{Broaden}

Constructs are shown in Table 2. Exercise self-efficacy scores appeared somewhat lower and disease management self-efficacy scores appeared somewhat higher than values from the literature. Patient activation scores 2 years post-intake revealed most were maintaining behaviors and pushing further; there appeared to be a much higher proportion is this highest stage of activation in the current sample.

\subsection{Build}

Heart-health behavior scores are shown in the top row of Supplemental Tables 1-4. At intake, 122 (52.8\%) participants were considered sufficiently active, $107(60.1 \%)$ at discharge, $102(65.0 \%)$ at 1 year and $87(56.9 \%)$ at 2 .

The bivariate associations between psychosocial well-being and health behaviors are reported in Supplemental Tables 1-4. First, with regard to exercise (Supplemental Table 1), it was related to greater selfregulation at intake and discharge across all subscales, although only a trend was observed for stimulus control. Greater exercise self-efficacy

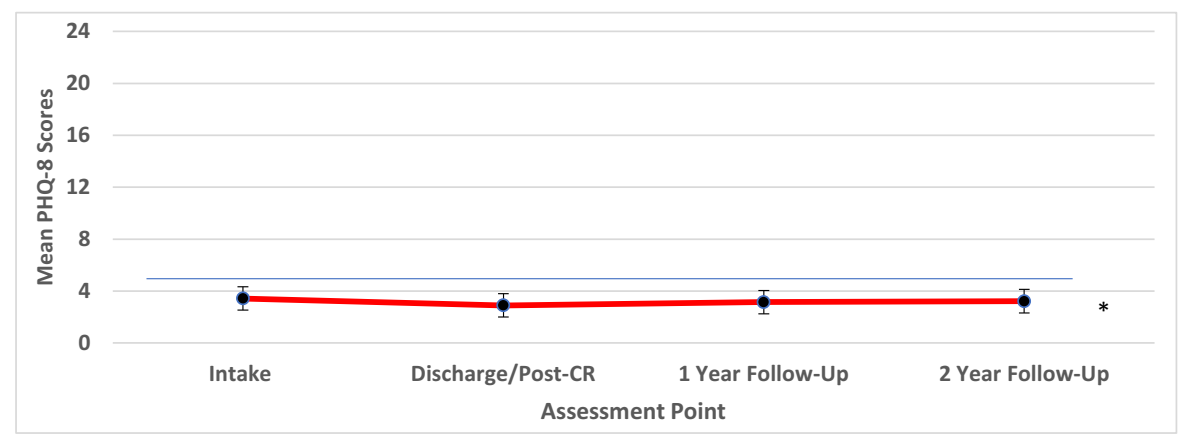

\section{A) Depressive symptoms}

Error bars.

General linear model for change over time: $\mathrm{F}=.85, \mathrm{p}=0.47$

$\mathrm{PHQ}=$ Patient Health Questionnaire; $\mathrm{CR}=$ cardiac rehabilitation.

*scores $<5$ indicate minimal depressive symptoms

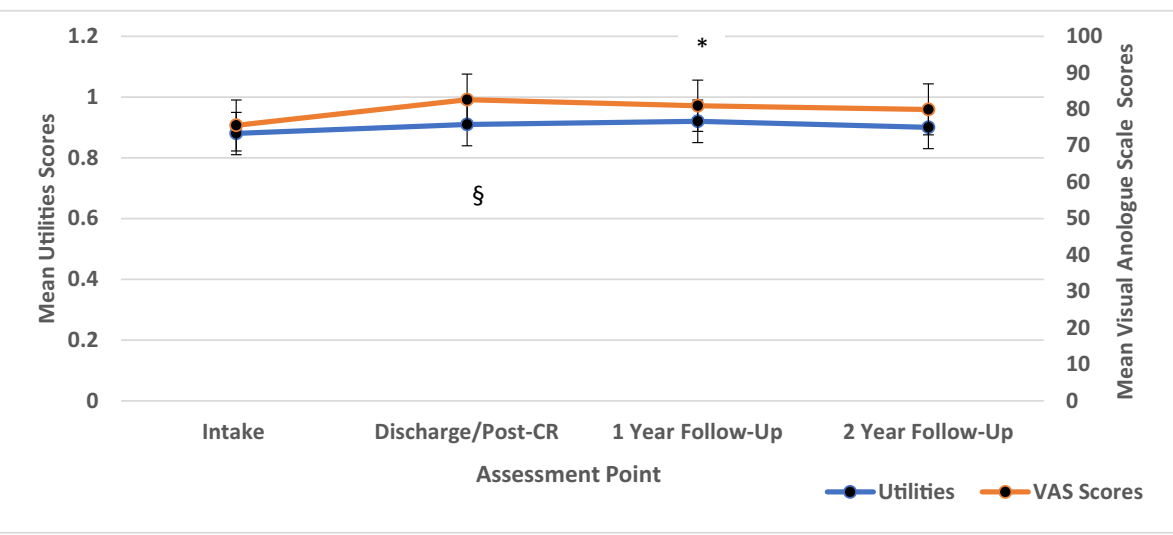

B) Quality of Life

Error bars.

General linear model for change over time for utilities: $\mathrm{F}=2.67, \mathrm{p}<0.05$; for VAS: $\mathrm{F}=5.08$, $\mathrm{p}<0.01$.

VAS $=$ Visual Analogue Scale; $\mathrm{CR}=$ cardiac rehabilitation.

*difference from intake to 1 year, $\mathrm{p}=0.05$ (trend).

$\S$ difference from intake to discharge, $\mathrm{p}=0.01$.

Fig. 3. Depressive symptoms and quality of life across the two years post-cardiac event/procedure in retained participants. 
was associated with greater exercise behavior at all points, as was greater chronic disease management efficacy at intake and 2 years. With regard to psychological well-being, greater exercise behavior was significantly related to greater self-acceptance at intake, as well as more environmental mastery, self-acceptance, positive relations and personal growth at 2 years. Greater exercise behavior was also significantly associated with greater resources through family and friends (intake) as well as one's neighborhood (intake and discharge). Finally, greater exercise behavior was associated with lower depressive symptoms at all assessment points (trends at discharge and 1 year), and greater QoL at all points (depending on indicator). Some other trends were observed.

With regard to nutrition (Supplemental Table 2), better hearthealthy eating was associated with greater exercise self-efficacy at all points. With regard to psychological well-being, better nutrition was significantly associated with greater self-acceptance at 1 and 2 years follow-up, as well as more positive relations at 2 years. With regard to chronic illness resources, significantly better nutrition was associated with greater overall, family and friend (also significant at discharge), neighborhood, organizational and community resources at 2 years and work resources at 1 year. Some other trends were observed. Greater nutrition was also significantly related to lower depressive symptoms and greater QoL at intake and discharge (other trends observed).

With regard to medication adherence (Supplemental Table 3), greater adherence was significantly associated with greater psychological well-being at 2 years in the domains of environmental mastery (actual and ideal), as well as autonomy and self-acceptance (actual). It was also associated with lower depressive symptoms at 1 and 2 years and greater QoL at 1 year. Again, some other trends were observed.

With regard to smoking status (Supplemental Table 4), it was significantly associated with the self-regulation subscale of behavioral monitoring at intake and stimulus control at discharge. Post-hoc tests revealed that non-smokers had greater behavioral monitoring $(p<.01)$ and stimulus control $(p<.01)$ than smokers. Finally, with regard to chronic illness resources, there was a significant association between smoking and work resources at all assessment points across the 2 years of study. Post-hoc tests revealed that non-smokers perceived greater work resources at CR intake $(p<.01)$, discharge $(p \leq .01)$, 1 year $(p<.01)$, and 2 years $(p<.01)$ than previous smokers. Some other trends for resources were observed, but no associations for selfefficacy, depressive symptoms or QoL.

Finally, the association between the following psychosocial wellbeing indicators and heart-health behaviors was maintained after adjusting for covariates: self-regulation, self-efficacy, some psychological well-being subscales and depressive symptoms (Table 3). As shown, greater self-regulation was significantly associated with greater exercise at intake. Greater exercise self-efficacy was significantly associated with better nutrition and greater exercise at 1 year; greater chronic disease self-efficacy was significantly associated with greater exercise at 2 years. With regards to psychological well-being, greater environmental mastery (actual) was significantly associated with greater exercise at 2 years. Finally, lower depressive symptoms were significantly associated with better diet at 2 years. Overall, after adjustment, psychosocial well-being indicators were associated with diet and exercise, but not medication adherence or smoking.

\subsection{Gains}

Similar to other samples, participants perceived their greatest resource in managing their CVD were their healthcare providers, but those who were employed in this sample also reported perceiving great resources in the work setting (particularly in relation to other samples as well; Table 2). Fig. 3B displays QoL across the 2 years in the retained sample, respectively. QoL scores were very high, and significantly increased from intake.

Work status is displayed in Fig. 4. At intake, the concordance between participants' desired and actual work status was $63.3 \%$ (Cohen's kappa). Post-CR, the concordance was 69.7\%, with $122(51.7 \%)$ retired and 119 (50.9\%) desiring to be retired. Only 18 (7.9\%) participants reported difficulty working at their paid employment because of their cardiovascular health at discharge.

\section{Discussion}

There have been few studies examining the trajectory of psychosocial well-being in cardiac patients [34]. Here we found CR patients reported significant personal growth, and were at the highest stage of behavioral activation, as reflected in their positive heart-health behaviors (although $40 \%$ of patients did not accrue the recommended 150 min of moderate to vigorous-intensity physical activity at 2 years). They also, on average, experienced very minimal depressive symptoms and high QoL consistently across the 2 years. Just over half of CR patients were retired as desired, and employed patients derived many resources from work to support their CVD self-management. As hypothesized, multiple indicators of psychosocial well-being were associated with restorative heart-health behaviors, specifically diet and exercise (but not medication adherence), over 2 years. Overall, results of this study suggest that CR patients are on average coping quite well with one of the most common chronic diseases, and those with positive psychosocial well-being may benefit from better heart-health behavior, and hence potentially cardiac outcomes.

On the whole, CR patients had quite good self-regulation [31], selfefficacy [22,23] and activation [32] to undertake heart-health behaviors. Few patients reported their CVD hampered their ability to work. While their environmental mastery was quite high, patients did desire greater mastery suggesting their CVD could be limiting them in some way. However, patients reported greater personal growth, positive relations with others, purpose in life and self-acceptance than desired (how this should be interpreted warrants further study, perhaps through qualitative inquiry). Healthcare providers were one of the greatest resources in supporting patients to manage their CVD.

It was curious that a significant reduction in depressive symptoms was not observed following the initial assessment point, as it is generally established that patients do experience elevated distress at the time of a cardiac event or procedure [5]. The lack of significant change could be due to the fact that the initial assessment in this study occurred some weeks after hospital discharge, and hence symptoms may have already subsided as patients returned home and recovered.

The results of this study demonstrating an association between psychosocial well-being and the heart-health behaviors are generally consistent with the published studies in this area (see [9] for a review of studies related to cardiovascular health; note these associations have not been tested often in cardiac patients and hence this work represents an important contribution to the literature), and the Broaden and Build theory [15]. Associations between heart-health behaviors and some specific psychological well-being indicators have been observed previously, particularly for diet and exercise, although there are mixed findings. Medication adherence has not generally been considered [9], and therefore this study contributes to the literature that there may not be a strong association with well-being, although replication is warranted.

Contrarily the results of this study suggesting no sustained association between psychosocial well-being and smoking are not consistent with the literature which suggests greater well-being is associated with a reduced risk of smoking [9]. However, there are mixed findings in the literature with regard to eudaimonic well-being in particular, which was one of the key constructs assessed herein. There were few smokers in the current sample, and therefore the tests of association may have been under-powered. The addictive nature of cigarettes may outweigh any positive psychological impacts. Overall, further research on the association between psychosocial well-being and medication adherence (particularly depressive symptoms and QoL) as well as smoking (particularly self-regulation and resources) is warranted. 
Table 3

Adjusted $^{\mathrm{a}}$ association between psychosocial well-being indicators and heart-health behaviors ${ }^{\mathrm{b}}$.

\begin{tabular}{|c|c|c|c|c|c|}
\hline F (assessment point) & Overall model $^{\mathrm{c}}$ & Exercise & Diet & Medication adherence $^{\mathrm{d}}$ & Smoking \\
\hline Index of self-regulation (intake) & $2.69^{* *}$ & $7.52^{* *}$ & $<0.01$ & 0.10 & 2.80 \\
\hline Exercise self-efficacy ( 1 year) & $5.40^{\text {***}}$ & $8.47^{* *}$ & $14.60^{* * * *}$ & 0.18 & 1.63 \\
\hline Self-efficacy for managing chronic disease (2 years) & $2.65^{* *}$ & $7.49^{* *}$ & 1.90 & 0.10 & 1.29 \\
\hline \multicolumn{6}{|l|}{ Psychological well-being ( 2 years) } \\
\hline Environmental mastery (actual) & $3.39 * *$ & $7.44^{* *}$ & $<0.01$ & 1.68 & 2.85 \\
\hline Autonomy (actual) & 1.91 & & & & \\
\hline Self-acceptance (actual) & $2.67^{* *}$ & $4.19^{*}$ & 2.17 & 0.01 & 2.57 \\
\hline Positive relations (actual) & $3.02^{* *}$ & $5.11^{*}$ & $4.05^{*}$ & $<0.01$ & 0.37 \\
\hline Purpose in life (actual) & 0.68 & & & & \\
\hline Personal growth (actual) & 1.65 & & & & \\
\hline Patient activation ( 2 years) & $2.05^{*}$ & 0.86 & 0.01 & 0.67 & $3.62 *$ \\
\hline Chronic illness resource survey ( 2 years) & 1.54 & & & & \\
\hline Depressive symptoms (2 years) & $4.51^{* * * *}$ & $5.09 *$ & $7.09^{* * *}$ & 0.03 & 2.82 \\
\hline Quality of life (2 years) & 1.41 & 1.49 & 2.86 & 0.31 & 1.30 \\
\hline
\end{tabular}

${ }^{\text {a }}$ Models adjusted for age, sex, ethnocultural background and cardiac indication (i.e., revascularization vs no). The latter 2 were included in the models as there was retention bias in relation to these characteristics.

b The health behavior values from the same time point were used in the models.

c Values for health behaviors only shown where overall model is significant. Each row displays one model.

$\mathrm{d}$ The Morisky Medication Adherence Scale is protected by US and international trademark and copyright laws. Permission for use is required. A license agreement is available from: MMAS Research LLC, 14725 NE 20th St., Bellevue WA 98007.

${ }^{*} p<.05$ (trend).

** $p<.01$.

*** $p<.001$

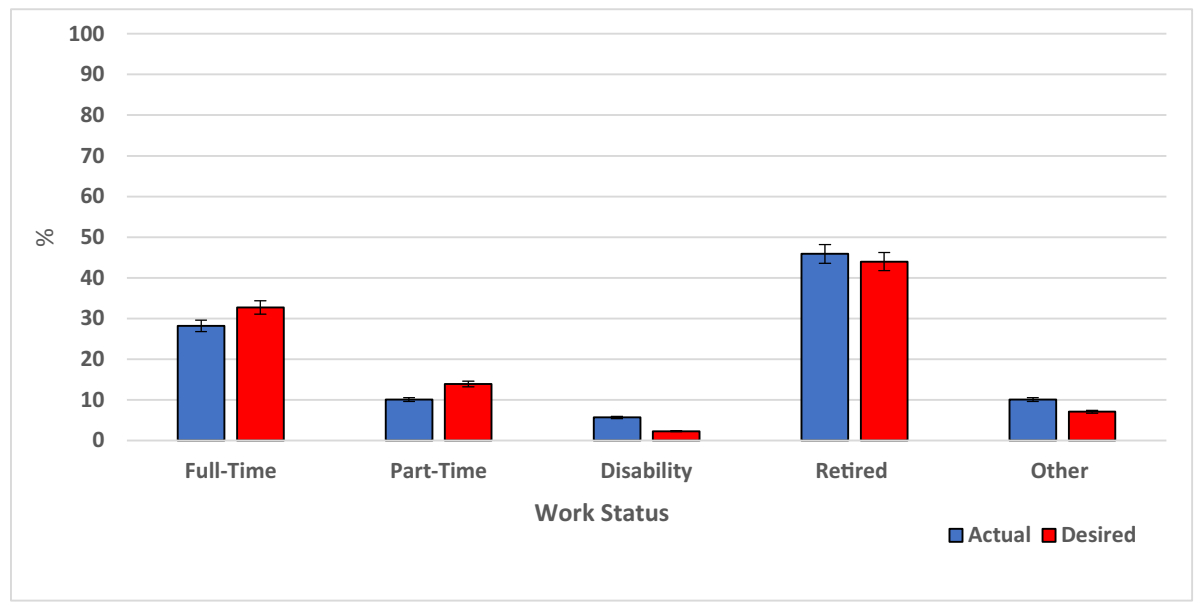

A) Cardiac Rehabilitation Intake, $\mathrm{N}=309$

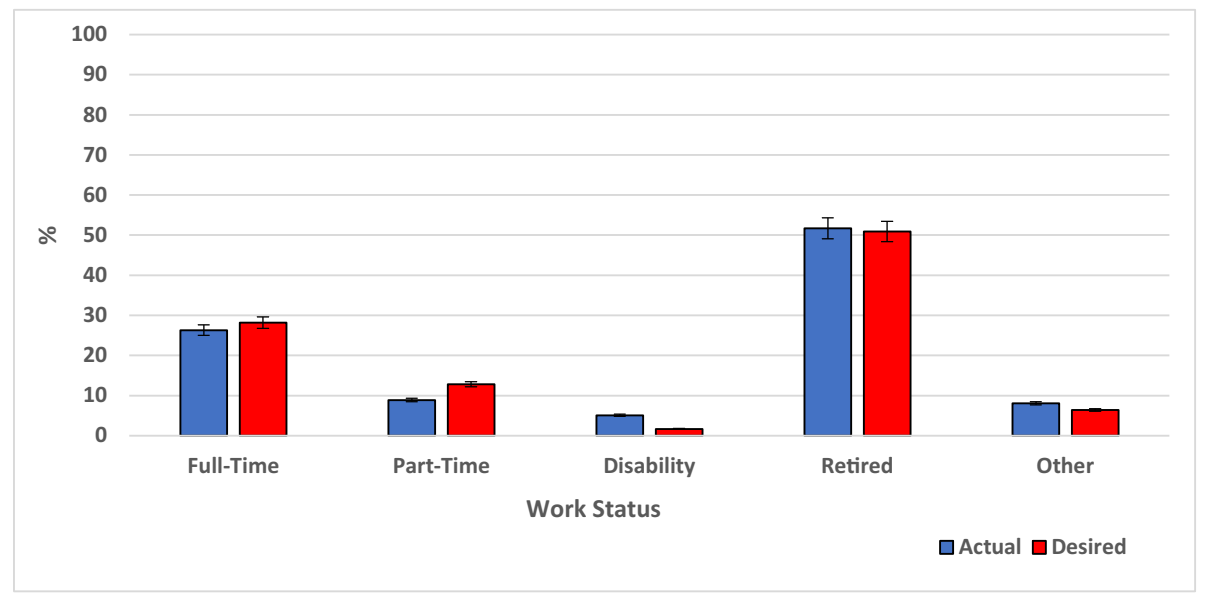

B) Discharge / Post-CR, N=234

Fig. 4. Participant work status (actual vs desired) by time. 
Several limitations to this study exist. First, there may be selection bias, as it is not ethical to collect characteristics on non-consenting patients and consent rate was not tracked. Second, there may be some retention bias in the sample, and there was much loss to follow-up. A notable number of patients consented to the study but then failed to complete the initial survey. Of most concern was that the sample retained at 2 years reported less depressive symptoms and better quality of life at baseline, as well as engaged in more exercise and consumed a more heart-healthy diet than those lost to follow-up. Moreover, the high degree of loss to follow-up precluded growth curve modeling to explore trajectories of psychosocial well-being and to fully test the Broaden and Build theory (note the mismatch of construct assessment timing in some instances also precluded a full test of the theory) [15]. This should be undertaken in future research. Third, generalizability is further limited to patients who have the resources and motivation to access CR, who are likely a more well-adjusted subgroup of patients than the average. While CR is the standard of care for this population, only approximately $30 \%$ of patients access it [35]. Moreover, patient's psychosocial wellbeing can be augmented through participation in CR [36,37]. Programs offer exercise training, patient education, psychological screening and stress management, support in return-to-work, among other core components, which all can ameliorate well-being [38]. Patients receive support from the multidisciplinary $\mathrm{CR}$ team and their peers. Indeed those retained at 2 years were more likely to have completed CR than those lost to follow-up. Future research is needed to characterize the well-being of all cardiac patients, as well-being may be greater in this cohort.

Fourth, heart-health behaviors were assessed via self-report, which invites social desirability bias. Fifth, multiple comparisons were performed, increasing the chance of type 1 error. A more conservative $p$ value was applied to mitigate this limitation. In addition, adjusted models were computed and the results of the models were considered more heavily in interpretation than the bivariate associations. Sixth, causal conclusions cannot be drawn from this study, due to the observational design. In particular, there may be a bi-directional association between psychosocial well-being and heart-health behaviors. Finally, optimism, life satisfaction and hedonic well-being are also key indicators of positive psychological well-being, but were not assessed in this study in an effort to minimize survey completion burden.

The implications of this research are that despite their chronic disease, cardiac patients can lead high-quality lives in the years following diagnosis; this is likely particularly true for those who participate in CR. There have been some interventions rigorously developed and empirically-validated in the literature to augment psychological well-being, which may further enhance health for all cardiac patients [39,40]. Unfortunately these interventions do not seem to be developed based on theory, although the rationale provided by Huffman et al. is highly concordant with the framework described in Fig. 1 [41]. Interestingly though, a positive psychological intervention developed by Huffman et al. for diabetes patients to promote lifestyle behavior change is based on a highly-testable framework. It warrants adaptation and testing in cardiac patients to determine whether it may be more applicable than Broaden-and-Build [42]. Whether such interventions can improve heart-healthy behaviors also warrants further study in the cardiac population [34,43]. It is possible that by broadening and building wellbeing, significant psychological distress $[44,45]$ and cardiovascular recurrences [46] could be mitigated.

\section{Conclusion}

In conclusion, $\mathrm{CR}$ initiators are thriving quite consistently over the course of their recovery trajectory, comparably to patients with other chronic diseases. Self-regulatory ability, self-efficacy, and low depressive symptoms were associated with the heart-health behaviors of diet and exercise, as was psychological/eudaimonic well-being (most notably self-acceptance and positive relations). Given the established association between psychosocial well-being and health outcomes in cardiac patients, the results of this study suggest many cardiac patients, particularly those that access CR, will have excellent quality and quantity of life for many years.

\section{Acknowledgments}

We would like to acknowledge Terry Fair, BEd, Cynthia Parson BSc, PT, RPT, and Ann Briggs, BSc, PT, RPT, for facilitating patient recruitment. We also acknowledge Gabriela Ghisi, Tomasz Kowal, Deborah Somanader, Marco Tomei, Yongyao Tan and Nickan Modamedi for their contributions to data collection and management. We are grateful for the statistical advice provided by Mirka Ondrak. The Morisky Medication Adherence Scale is protected by US and international trademark and copyright laws. Permission for use is required. A license agreement is available from: MMAS Research LLC, 14725 NE 20th St., Bellevue WA 98007.

\section{Conflict of interest}

The authors declare that they have no conflict of interest.

\section{Ethical approval}

All procedures performed in this study involving human participants were in accordance with the ethical standards of the institutional research committees and with the 1964 Helsinki declaration and its later amendments or comparable ethical standards.

\section{Informed consent}

Informed consent was obtained from all individual participants included in the study.

\section{Appendix A. Supplementary data}

Supplementary data to this article can be found online at https:// doi.org/10.1016/j.genhosppsych.2018.03.006.

\section{References}

[1] Mendis S, Puska P, Norrving B. Global Atlas on cardiovascular disease prevention and control. Geneva, Switzerland: World Health Organization; 2011. doi:NLM classification: WG 120.

[2] Thombs B, Bass E, Ford D, Steward K, Tsilidis K, Fauerbach J, et al. Prevalence of depression in survivors of acute myocardial infarction. J Gen Intern Med 2006;21:30-8.

[3] Pogosova NV, Saner H, Pedersen SS, Cupples ME, Mc Gee H, Hofer S, et al. Psychosocial aspects in cardiac rehabilitation: from theory to practice. A position paper from the Cardiac Rehabilitation Section of the European Association of Cardiovascular Prevention and Rehabilitation of the European Society of Cardiology. Kardiologiya 2015;55:96-108. http://dx.doi.org/10.1177/ 2047487314543075.

[4] Lichtman JH, Froelicher ES, Blumenthal JA, Carney RM, Doering LV, Frasure-Smith $\mathrm{N}$, et al. Depression as a risk factor for poor prognosis among patients with acute coronary syndrome: systematic review and recommendations: a scientific statement from the American Heart Association. Circulation 2014;129:1350-69. http://dx. doi.org/10.1161/CIR.0000000000000019.

[5] Grace SL, Abbey SE, Pinto R, Shnek ZM, Irvine J, Stewart DE. Longitudinal course of depressive symptomatology after a cardiac event: effects of gender and cardiac rehabilitation. Psychosom Med 2005;67:52-8. http://dx.doi.org/10.1097/01.psy. 0000151486.28349.70.

[6] Leung YW, Gravely-Witte S, Macpherson A, Irvine J, Stewart DE, Grace SL. Posttraumatic growth among cardiac outpatients: degree comparison with other chronic illness samples and correlates. J Health Psychol 2010;15:1049-63. http://dx.doi. org/10.1177/1359105309360577.

[7] Dubois CM, Vesga O, Beale EE, Healy BC, Boehm JK, Huffman JC. Relationships between positive psychological constructs and health outcomes in patients with cardiovascular disease: a systematic review. Int J Cardiol 2015;195:265-80.

[8] Dubois CM, Beach SR, Kashdan TB, Nyer MB, Park ER, Celano CM, et al. Positive psychological attributes and cardiac outcomes: associations, mechanisms, and interventions. Psychosomatics 2012;53:303-18.

[9] Boehm JK, Kubzansky LD. The heart's content: the association between positive 
psychological well-being and cardiovascular health. Psychol Bull 2012;138:655-91.

[10] Scheier MF, Matthews KA, Owens JF, Magovern Sr GJ, Lefebvre RC, Abbott RA, et al. Dispositional optimism and recovery from coronary artery bypass surgery: the beneficial effects on physical and psychological well-being. J Pers Soc Psychol 1989;57:1024-40.

[11] Shepperd JA, Maroto JJ, Pbert LA. Dispositional optimism as a predictor of health changes among cardiac patients. J Res Pers 1996;30:517-34. http://dx.doi.org/10. 1006/jrpe.1996.0038.

[12] Tindle H, Belnap BH, Houck PR, Mazumdar S, Scheier MF, Matthews KA, et al Optimism, response to treatment of depression, and rehospitalization after coronary artery bypass graft surgery. Psychosom Med 2014;74:200-7. http://dx.doi.org/10. 1097/PSY.0b013e318244903f.

[13] Giltay EJ, Geleijnse JM, Zitman FG, Buijsse B, Kromhout D. Lifestyle and dietary correlates of dispositional optimism in men: the Zutphen elderly study. J Psychosom Res 2007;63:483-90. http://dx.doi.org/10.1016/j.jpsychores.2007.07.014.

[14] Steptoe A, Dockray S, Wardle J. Positive affect and psychobiological processes relevant to health. Epidemiology 2010;77:1-19. http://dx.doi.org/10.1111/j.14676494.2009.00599.x.Positive.

[15] Fredrickson BL. The broaden-and-build theory of positive emotions. Philos Trans R Soc Lond B Biol Sci 2004;359:1367-78.

[16] Grace SL, Tan Y, Marcus L, Dafoe W, Simpson C, Suskin N, et al. Perceptions of cardiac rehabilitation patients, specialists and rehabilitation programs regarding cardiac rehabilitation wait times. BMC Health Serv Res 2012;12:259http://dx.doi. org/10.1186/1472-6963-12-259.

[17] Somanader D, Chessex C, Ginsburg L, Grace SL. The quality and variability of cardiac rehabilitation delivery. J Cardiopulm Rehabil Prev n.d.

[18] Stone J, Arthur H. Canadian guidelines for cardiac rehabilitation and cardiovascular disease prevention: Translating knowledge into action. 3rd ed Winnipeg, Manitoba, Canada: Canadian Association of Cardiac Rehabilitation (CACR; 2009.

[19] Spitzer RL, Kroenke K, Williams JB. Validation and utility of a self-report version of PRIME-MD: the PHQ primary care study. Primary care evaluation of mental disorders. Patient health questionnaire. JAMA 1999;282:1737-44.

[20] Ryff CD, Keyes CL. The structure of psychological well-being revisited. J Pers Soc Psychol 1995;69:719-27. http://dx.doi.org/10.1037/0022-3514.69.4.719.

[21] Fleury J. The index of self-regulation: development and psychometric analysis. J Nurs Meas 1998;6:3-17.

[22] Hickey ML, Owen SV, Froman RD. Instrument development: cardiac diet and exercise self-efficacy. Nurs Res 1992;41:347-51. (doi:1437584).

[23] Lorig KR, Sobel DS, Ritter PL, Laurent D, Hobbs M. Effect of a self-management program on patients with chronic disease. Eff Clin Pract 2001;4:256-62.

[24] Hibbard JH, Stockard J, Mahoney ER, Tusler M. Development of the patient activation measure (PAM): conceptualizing and measuring activation in patients and consumers. Health Serv Res 2004;39:1005-26. http://dx.doi.org/10.1111/j.1475 6773.2004.00269.x.

[25] Godin G, Shephard R. Godin leisure-time exercise questionnaire. Med Sci Sports Exerc 1985;29:36-8.

[26] Walker SN, Sechrist KR, Pender NJ. The health-promoting lifestyle profile: development and psychometric characteristics. Nurs Res 1987;36:76-81.

[27] Morisky DE, DiMatteo MR. Improving the measurement of self-reported medication nonadherence: response to authors. J Clin Epidemiol 2011;64:255-63.

[28] Herdman M, Gudex C, Lloyd A, Janssen M, Kind P, Parkin D, et al. Development and preliminary testing of the new five-level version of EQ-5D (EQ-5D-5L). Qual Life Res 2011;20:1727-36. http://dx.doi.org/10.1007/s11136-011-9903-x.

[29] Glasgow RE, Toobert DJ, Barrera M, Strycker LA. The chronic illness resources survey: cross-validation and sensitivity to intervention. Health Educ Res 2005;20:402-9. http://dx.doi.org/10.1093/her/cyg140.

[30] Ali S, Chessex C, Bassett-Gunter R, Grace S. Patient satisfaction with cardiac rehabilitation: association with utilization, functional capacity, and heart-health behaviors. Patient Prefer Adherence 2017. http://dx.doi.org/10.2147/PPA.S120464.
[31] Yeom H-A, Choi M, Belyea M, Fleury J. Psychometric evaluation of the index of selfregulation. West J Nurs Res 2011;33:268-85. http://dx.doi.org/10.1177/ 0193945910378854.

[32] Skolasky RL, Green AF, Scharfstein D, Boult C, Reider L, Wegener ST. Psychometric properties of the patient activation measure among multimorbid older adults. Health Serv Res 2011;46:457-78. http://dx.doi.org/10.1111/j.1475-6773.2010. 01210.x.

[33] Glasgow RE, Strycker LA, Toobert DJ, Eakin E. A social-ecologic approach to assessing support for disease self-management: the chronic illness resources survey. $\mathrm{J}$ Behav Med 2000;23:559-83. http://dx.doi.org/10.1023/A:1005507603901.

[34] Peterson C, Kim ES. Psychological interventions for coronary heart disease. Int J Clin Health Psychol 2011;11:563-75. http://dx.doi.org/10.1002/14651858. CD002902.pub3.

[35] Turk-Adawi K, Sarrafzadegan N, Grace SL. Global availability of cardiac rehabilitation. Nat Rev Cardiol 2014;11:586-96. http://dx.doi.org/10.1038/ nrcardio.2014.98.

[36] Lavie CJ, Milani RV, O'Keefe JH, Lavie TJ. Impact of exercise training on psychological risk factors. Prog Cardiovasc Dis 2011;53:464-70. http://dx.doi.org/10. 1016/j.pcad.2011.03.007.

[37] Roviaro R, Holmes DS, Holmsten RD. Influence of a cardiac rehabilitation program on the cardiovascular, psychological, and social functioning of cardiac patients. J Behav Med 1984;7:61.

[38] Grace SL, Turk-Adawi KI, Contractor A, Atrey A, Campbell NRC, Derman W, et al. Cardiac rehabilitation delivery model for low-resource settings: an international council of cardiovascular prevention and rehabilitation consensus statement. Prog Cardiovasc Dis 2016;59:303-22. http://dx.doi.org/10.1016/j.pcad.2016.08.004.

[39] Huffman JC, Mastromauro CA, Boehm JK, Seabrook R, Fricchione GL, Denninger JW, et al. Development of a positive psychology intervention for patients with acute cardiovascular disease. 2011;6. http://dx.doi.org/10.4081/hi.2011.e13.

[40] Sanjuán P, Montalbetti T, Pérez-García AM, Bermúdez J, Arranz H, Castro A. A randomised trial of a positive intervention to promote well-being in cardiac patients. Appl Psychol Health Well Being 2016;8:64-84. http://dx.doi.org/10.1111/ aphw.12062.

[41] Huffman JC, Millstein RA, Mastromauro CA, Moore SV, Celano CM, Bedoya CA, et al. A positive psychology intervention for patients with an acute coronary syndrome: treatment development and proof-of-concept trial. J Happiness Stud 2016;17:1985-2006. http://dx.doi.org/10.1007/s10902-015-9681-1.

[42] Huffman JC, DuBois CM, Millstein RA, Celano CM, Wexler D. Positive psychological interventions for patients with type 2 diabetes: rationale, theoretical model, and intervention development. J Diabetes Res 2015;2015:428349http://dx.doi.org/10. $1155 / 2015 / 428349$.

[43] Huffman JC, Albanese AM, Campbell KA, Celano CM, Millstein RA, Mastromauro $\mathrm{CA}$, et al. The positive emotions after acute coronary events behavioral health intervention: design, rationale, and preliminary feasibility of a factorial design study. Clin Trials 2017;14:128-39. http://dx.doi.org/10.1177/1740774516673365.

[44] Klainin-Yobas P, Ng SH, Stephen PDM, Lau Y. Efficacy of psychosocial interventions on psychological outcomes among people with cardiovascular diseases: a systematic review and meta-analysis. Patient Educ Couns 2016;99:512-21. http://dx.doi.org/ 10.1016/j.pec.2015.10.020.

[45] Nikrahan GR, Suarez L, Asgari K, Beach SR, Celano CM, Kalantari M, et al. Positive psychology interventions for patients with heart disease: a preliminary randomized trial. Psychosomatics 2016;57:348-58. http://dx.doi.org/10.1016/j.psym.2016.03. trial.

[46] Davidson KW, Rieckmann N, Clemow L, Schwartz JE, Shimbo D, Medina V, et al. Enhanced depression care for patients with acute coronary syndrome and persistent depressive symptoms: coronary psychosocial evaluation studies randomized controlled trial. Arch Intern Med 2010;170:600-8. 